\title{
Spin Structures of Textured and Isotropic Nd-Fe-B-Based Nanocomposites: Evidence for Correlated Crystallographic and Spin Textures
}

A. Michels, ${ }^{1,}{ }^{*}$ R. Weber, ${ }^{1}$ I. Titov, ${ }^{1}$ D. Mettus,${ }^{1}$ É. A. Périgo, ${ }_{7}^{1,2}$ I. Peral, ${ }^{1,3}$ O. Vallcorba, ${ }^{4}$ J. Kohlbrecher, ${ }^{5}$ K. Suzuki, ${ }^{6}$ M. Ito, ${ }^{7}$ A. Kato, ${ }^{7}$ and M. Yano ${ }^{7}$

${ }^{1}$ Physics and Materials Science Research Unit, University of Luxembourg, 162a avenue de la Faencerie, L-1511 Luxembourg, Luxembourg

${ }^{2}$ ABB Corporate Research Center, 940 Main Campus Drive, Raleigh, North Carolina 27606, USA

${ }^{3}$ Materials Research and Technology Department, Luxembourg Institute of Science and Technology, 41 rue du Brill, L-4422 Belvaux, Luxembourg

${ }^{4}$ Alba Synchrotron, Carrer de la Llum 2-26, 08290 Cerdanyola del Vallès, Barcelona, Spain

${ }^{5}$ Paul Scherrer Institute, $\mathrm{CH}-5232$ Villigen PSI, Switzerland

${ }^{6}$ Department of Materials Science and Engineering, Monash University, Clayton, Victoria 3800, Australia

${ }^{7}$ Advanced Material Engineering Division, Toyota Motor Corporation, Susono 410-1193, Japan

(Received 1 September 2016; revised manuscript received 1 December 2016; published 9 February 2017)

We report the results of a comparative study of the magnetic microstructure of textured and isotropic $\mathrm{Nd}_{2} \mathrm{Fe}_{14} \mathrm{~B} / \alpha$-Fe nanocomposites using magnetometry, transmission electron microscopy, synchrotron $\mathrm{x}$-ray diffraction, and, in particular, magnetic small-angle neutron scattering (SANS). Analysis of the magnetic neutron data of the textured specimen and computation of the correlation function of the spinmisalignment SANS cross section suggests the existence of inhomogeneously magnetized regions on an intraparticle nanometer length scale, about 40-50 nm in the remanent state. Possible origins for this spin disorder are discussed: it may originate in thin-grain-boundary layers (where the material parameters are different than in the $\mathrm{Nd}_{2} \mathrm{Fe}_{14} \mathrm{~B}$ grains), or it may reflect the presence of crystal defects (introduced via hot pressing), or the dispersion in the orientation distribution of the magnetocrystalline anisotropy axes of the $\mathrm{Nd}_{2} \mathrm{Fe}_{14} \mathrm{~B}$ grains. X-ray powder diffraction data reveal a crystallographic texture in the direction perpendicular to the pressing direction - a finding which might be related to the presence of a texture in the magnetization distribution, as inferred from the magnetic SANS data.

DOI: 10.1103/PhysRevApplied.7.024009

\section{INTRODUCTION}

Nd-Fe-B-based nanocomposite permanent magnets, which consist of exchange-coupled nanocrystalline hard $\left(\mathrm{Nd}_{2} \mathrm{Fe}_{14} \mathrm{~B}\right)$ and soft $\left(\alpha-\mathrm{Fe}\right.$ or $\left.\mathrm{Fe}_{3} \mathrm{~B}\right)$ magnetic phases, are of potential interest for electronic devices, motors, and wind turbines due to their preeminent magnetic properties such as high remanence and magnetic energy product [1-6]. The major challenge remains the understanding of how the features of the microstructure (e.g., average particle size and shape, volume fraction of the soft phase, texture, and interfacial chemistry) correlate with their magnetic properties. In order to tackle this issue, a multiscale characterization approach is adopted, which comprises a suite of both experimental and theoretical state-of-the-art methods such as high-resolution electron microscopy, electron backscattering diffraction, three-dimensional atom-probe analysis, Lorentz and Kerr microscopy, or atomistic and continuum micromagnetic simulations.

Recent investigations by Liu et al. [7] and Sepehri-Amin et al. [8] demonstrate that the properties of the interface regions between the $\mathrm{Nd}_{2} \mathrm{Fe}_{14} \mathrm{~B}$ grains decisively determine

*andreas.michels@uni.lu the coercivity of the sample. The grain-boundary layers (and triple junctions between the grains) have a thickness between about 1 and $15 \mathrm{~nm}$ and can be both in a crystalline or amorphous state. Moreover, as far as their magnetism is concerned, the intergranular regions are characterized by different magnetic interactions (exchange, magnetocrystalline anisotropy, and saturation magnetization) as compared to the $\mathrm{Nd}_{2} \mathrm{Fe}_{14} \mathrm{~B}$ crystallites, and, hence, they represent potential sources for the nucleation of inhomogeneous spin textures during magnetization reversal. Indeed, the micromagnetic simulation results reported in Ref. [8] suggest that the existence of a thin $(<5 \mathrm{~nm})$ ferromagnetic grainboundary phase with reduced magnetocrystalline anisotropy, exchange-stiffness constant, and saturation magnetization causes the magnetization reversal to occur from the soft intergranular phase into the hard $\mathrm{Nd}_{2} \mathrm{Fe}_{14} \mathrm{~B}$ phase at a field of $-2.5 \mathrm{~T}$. When the intergranular phase is nonmagnetic, the nucleation of reversed domains starts from the triple junctions of the $\mathrm{Nd}_{2} \mathrm{Fe}_{14} \mathrm{~B}$ grains at a higher field of $-3.2 \mathrm{~T}$.

In this context, it also worth noting that first-principles density-functional-theory calculations on an exchangespring multilayer system [9] predict a dependence of the exchange coupling on the crystallographic orientation at the interface between $\mathrm{Nd}_{2} \mathrm{Fe}_{14} \mathrm{~B}$ and $\alpha$-Fe; specifically, 
ferromagnetic coupling is predicted for the $\mathrm{Nd}_{2} \mathrm{Fe}_{14} \mathrm{~B}(001) / \alpha-\mathrm{Fe}(001)$ interface model, whereas antiferromagnetic interactions are obtained for $\mathrm{Nd}_{2} \mathrm{Fe}_{14} \mathrm{~B}(100) / \alpha-\mathrm{Fe}(110)$. If this prediction is true, then it may negatively influence the magnetic properties of this class of materials (e.g., the maximum-energy product), and it may become more difficult to realize a particular application (e.g., in a motor). Indeed, a recent experimental study using ferromagnetic resonance and Kerr microscopy [10] reports on the predicted negative exchange coupling between a 10-nm-thick $\alpha$-Fe layer and the surface of a $\mathrm{Nd}_{2} \mathrm{Fe}_{14} \mathrm{~B}$ single crystal.

The above-discussed examples ultimately demonstrate that the magnetic microstructure of nanocrystalline $\mathrm{Nd}-\mathrm{Fe}-\mathrm{B}-$ based magnets is characterized by inhomogeneous magnetization structures and that interfacial regions are a major cause for the nanoscale spin disorder. In addition to the grain boundaries, there exist, however, other sources of spin disorder in such materials: ultrafinegrained textured nanocomposites are produced from melt-spun ribbons via hot deformation [7,8,11,12]; this process may introduce crystal defects which locally act as nucleation centers for nonuniform magnetization textures. Furthermore, one has to invoke a magnetization inhomogeneity which is due to the nonideal alignment (dispersion) of the crystallographic $c$ axes (of the $\mathrm{Nd}_{2} \mathrm{Fe}_{14} \mathrm{~B}$ grains) along the pressing direction during hot deformation; the spins have to undergo rotations in order to accommodate to the changes in the easy-axis magnetization direction from grain to grain. Last but not least, there is the magnetic shape anisotropy of the usually platelet-shaped $\mathrm{Nd}_{2} \mathrm{Fe}_{14} \mathrm{~B}$ particles, which may result in a small spin canting towards the plane perpendicular to the $c$ axis. It is certainly true that the magnetic anisotropy field of the $\mathrm{Nd}_{2} \mathrm{Fe}_{14} \mathrm{~B}$ phase (about $8 \mathrm{~T}$ at $300 \mathrm{~K} \mathrm{[13])} \mathrm{is} \mathrm{much} \mathrm{larger} \mathrm{than} \mathrm{any} \mathrm{shape-anisotropy}$ field (assuming, e.g., $0.5 \mathrm{~T}$ for strongly anisotropic grains), but, nevertheless, weak spin canting $\left[\tan ^{-1}(0.5 / 8) \cong 3.6^{\circ}\right]$ might be produced by the competition between shape and magnetocrystalline anisotropy.

In order to scrutinize the above-described issue-the interplay between microstructural defects and nonuniform spin structures in Nd-Fe-B-based nanocompositeswe carry out a comparative study of the magnetic microstructure of textured (hot-deformed) and isotropic nanocrystalline $\mathrm{Nd}_{2} \mathrm{Fe}_{14} \mathrm{~B} / \alpha$ - $\mathrm{Fe}$ by means of magnetic small-angle neutron scattering (SANS). Specifically, the central aim of our investigation is to detect and quantify the presumed nanoscale spin disorder in these materials, which is commonly only indirectly inferred by combining (locally observed) results from electron microscopy, atom-probe analysis, magnetization, and micromagnetic simulations. The understanding of the details of the microscopic spin distribution of Nd-Fe-B-based nanocrystalline permanent magnets is of importance, since this provides the required link to the understanding of their overall macroscopic magnetic properties (e.g., the coercivity or the exchange coupling mechanism). In this respect, the neutron-dataanalysis procedure that is reported in this paper may provide important insights for the further development of magnetic materials with a higher magnetic flux.

The SANS technique (see Ref. [14] for a review) provides information about the variations of both the magnitude and orientation of the magnetization vector field in the bulk and on a nanometer length scale (approximately 1-300 $\mathrm{nm}$ ). SANS is extremely sensitive to longwavelength magnetization fluctuations, and it has only recently been employed for characterizing $\mathrm{Nd}-\mathrm{Fe}-\mathrm{B}-$ based permanent magnets: for example, the field dependence of characteristic magnetic length scales during the magnetization-reversal process in isotropic Nd-Fe-B-based nanocomposites [15] and in isotropic sintered Nd-Fe-B [16] was studied, the exchange-stiffness constant has been determined [17], the observation of the so-called spike anisotropy in the magnetic SANS cross section has been explained with the formation of flux-closure patterns [18], magnetic multiple scattering has been detected [19], textured Nd-Fe-B has been investigated [20], and the effect of grain-boundary diffusion on the magnetization-reversal process of isotropic [21] and hot-deformed textured [22-24] nanocrystalline Nd-Fe-B magnets has been studied. In this paper, exchange-coupled model magnets are investigated by SANS in order to quantitatively evaluate the spin disorder and to clarify the possible anisotropy of magnetic interactions.

The paper is organized as follows: Sec. II provides the details of the sample characterization and of the neutron experiment. Section III discusses the unpolarized SANS cross sections for the perpendicular and parallel scattering geometries; the differences regarding the expected angular anisotropies are highlighted. Section IV presents and discusses the experimental results of the magnetization, electron microscopy, x-ray synchrotron, and neutron measurements. Section V summarizes the main findings of this study.

\section{EXPERIMENT}

Two $\mathrm{Nd}_{2} \mathrm{Fe}_{14} \mathrm{~B} / \alpha$-Fe nanocomposites containing, respectively, $5 \mathrm{wt} \%$ of $\alpha$-Fe are investigated in this study. Both samples are prepared by means of the melt-spinning technique. One sample is subsequently hot deformed in order to obtain a textured magnet. For this purpose, the melt-spun ribbons are crushed into powders of a few hundred micrometers in size and then sintered at $973 \mathrm{~K}$ under a pressure of $400 \mathrm{MPa}$. The sintered bulk is hot deformed with a height reduction of about $75 \%$ to develop the [001] texture of the $\mathrm{Nd}_{2} \mathrm{Fe}_{14} \mathrm{~B}$ phase along the pressing direction $[8,12]$. This results in the formation of plateletshaped $\mathrm{Nd}_{2} \mathrm{Fe}_{14} \mathrm{~B}$ grains with an average thickness of approximately $110 \mathrm{~nm}$ and an average diameter of approximately $140 \mathrm{~nm}$. The $\mathrm{Nd}_{2} \mathrm{Fe}_{14} \mathrm{~B}$ platelets are stacked along 


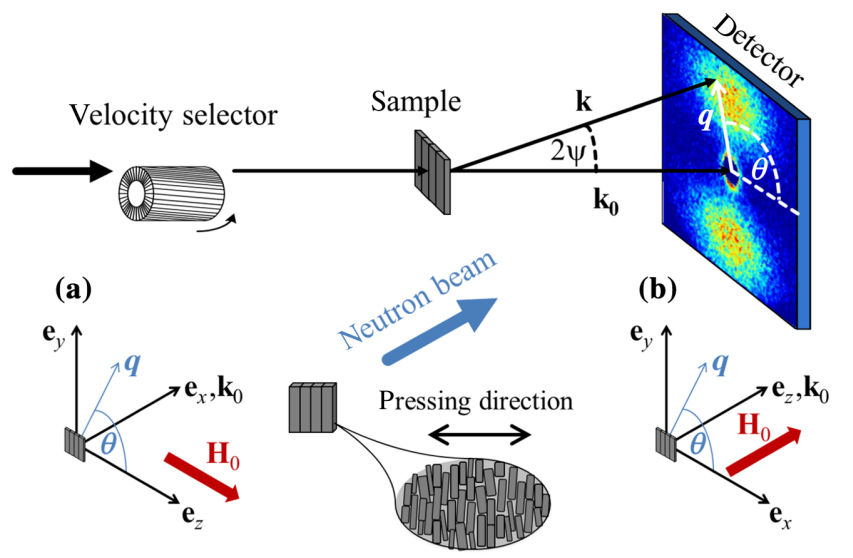

FIG. 1. Sketch of the perpendicular (a) and parallel (b) scattering geometry, which, respectively, have the applied magnetic field $\mathbf{H}_{0}$ perpendicular and parallel to the wave vector $\mathbf{k}_{0}$ of the incident neutron beam; $q=|\mathbf{q}|=4 \pi \lambda^{-1} \sin \psi$, where $2 \psi$ denotes the scattering angle, and $\lambda$ is the mean neutron wavelength. Note that $\mathbf{H}_{0} \| \mathbf{e}_{z}$ in both geometries and that $\mathbf{q} \cong\left(0, q_{y}, q_{z}\right)=$ $q(0, \sin \theta, \cos \theta)$ for $\mathbf{k}_{0} \perp \mathbf{H}_{0} \quad$ (a) and $\mathbf{q} \cong\left(q_{x}, q_{y}, 0\right)=$ $q(\cos \theta, \sin \theta, 0)$ for $\mathbf{k}_{0} \| \mathbf{H}_{0}$ (b). The pressing direction is horizontal, which is along $\mathbf{e}_{z}$ in (a) and along $\mathbf{e}_{x}$ in (b).

the nominal $c$ axis, which we define as the [001] direction, with some degrees of misorientation (see below). The isotropic sample has an average grain size of about $20 \mathrm{~nm}$. We also investigate composites with 0 and $10 \mathrm{wt} \%$ of $\mathrm{Fe}$, which, as far as the neutron results are concerned, show qualitatively the same behavior as the 5-wt \% sample. For further details, see Refs. [22-24].

The neutron experiment is carried out at $300 \mathrm{~K}$ at the instrument SANS-I at the Paul Scherrer Institute, Switzerland, using unpolarized neutrons with a mean wavelength of $\lambda=4.5 \AA$ and $\Delta \lambda / \lambda=10 \%$ (FWHM) $[25,26]$. The external magnetic field $\mathbf{H}_{0}$ (provided by a cryomagnet; $\mu_{0} H_{\max }=9.5 \mathrm{~T}$ ) is applied perpendicular and parallel to the wave vector $\mathbf{k}_{0}$ of the incoming neutron beam (compare Fig. 1); this corresponds to the situation that $\mathbf{H}_{0}$ is parallel $\left(\mathbf{k}_{0} \perp \mathbf{H}_{0}\right)$ and perpendicular $\left(\mathbf{k}_{0} \| \mathbf{H}_{0}\right)$ to the nominal $c$ axis (pressing direction) of the textured sample. Neutron data are corrected for background scattering (empty sample holder), transmission, and detector efficiency using the GRASP software package [27]. The measured transmission is larger than $90 \%$ for both samples at all fields investigated. Further sample characterization is done by means of vibrating sample magnetometry, transmission electron microscopy, and synchrotron x-ray diffraction (at beam line BL04-MSPD at the ALBA Synchrotron, Barcelona, Spain [28]).

\section{UNPOLARIZED SANS CROSS SECTIONS AND CORRELATION FUNCTION}

The elastic unpolarized SANS cross section $d \Sigma / d \Omega$ at momentum-transfer vector $\mathbf{q}$ takes on different forms depending on the relative orientation between the wave vector $\mathbf{k}_{0}$ of the incident neutron beam and the externally applied magnetic field $\mathbf{H}_{0}$ [14]; for the perpendicular geometry $\left(\mathbf{k}_{0} \perp \mathbf{H}_{0}\right)$, we obtain

$$
\begin{aligned}
\frac{d \Sigma}{d \Omega}(\mathbf{q})= & \frac{8 \pi^{3}}{V}\left(|\tilde{N}|^{2}+b_{H}^{2}\left|\tilde{M}_{x}\right|^{2}+b_{H}^{2}\left|\tilde{M}_{y}\right|^{2} \cos ^{2} \theta\right. \\
& +b_{H}^{2}\left|\tilde{M}_{z}\right|^{2} \sin ^{2} \theta-b_{H}^{2}\left(\tilde{M}_{y} \tilde{M}_{z}^{*}+\tilde{M}_{y}^{*} \tilde{M}_{z}\right) \\
& \times \sin \theta \cos \theta),
\end{aligned}
$$

whereas for the parallel case $\left(\mathbf{k}_{0} \| \mathbf{H}_{0}\right)$,

$$
\begin{aligned}
\frac{d \Sigma}{d \Omega}(\mathbf{q})= & \frac{8 \pi^{3}}{V}\left(|\tilde{N}|^{2}+b_{H}^{2}\left|\tilde{M}_{x}\right|^{2} \sin ^{2} \theta+b_{H}^{2}\left|\tilde{M}_{y}\right|^{2} \cos ^{2} \theta\right. \\
& \left.+b_{H}^{2}\left|\tilde{M}_{z}\right|^{2}-b_{H}^{2}\left(\tilde{M}_{x} \tilde{M}_{y}^{*}+\tilde{M}_{x}^{*} \tilde{M}_{y}\right) \sin \theta \cos \theta\right)
\end{aligned}
$$

$V$ denotes the scattering volume, $b_{H}=2.91 \times 10^{8} \mathrm{~A}^{-1} \mathrm{~m}^{-1}$, $\tilde{N}(\mathbf{q})$ is the nuclear scattering amplitude, and $\tilde{\mathbf{M}}(\mathbf{q})=$ $\left\{\tilde{M}_{x}(\mathbf{q}), \tilde{M}_{y}(\mathbf{q}), \tilde{M}_{z}(\mathbf{q})\right\}$ represents the Fourier transform of the magnetization $\mathbf{M}(\mathbf{r})=\left\{M_{x}(\mathbf{r}), M_{y}(\mathbf{r}), M_{z}(\mathbf{r})\right\} ; c^{*}$ is a quantity complex conjugated to $c$. We emphasize that the magnetization vector field of a bulk ferromagnet is a function of the position $\mathbf{r}=\{x, y, z\}$ inside the material, i.e., $\quad \mathbf{M}=\mathbf{M}(x, y, z)$ and that, consequently, $\quad \tilde{\mathbf{M}}=$ $\tilde{\mathbf{M}}\left(q_{x}, q_{y}, q_{z}\right)$. However, the Fourier components which appear in the above SANS cross sections represent projections into the $q_{y}-q_{z}$ plane for $\mathbf{k}_{0} \perp \mathbf{H}_{0}\left(q_{x} \cong 0\right)$ and into the $q_{x}-q_{y}$ plane for $\mathbf{k}_{0} \| \mathbf{H}_{0}\left(q_{z} \cong 0\right)$ (compare Fig. 1). In polar coordinates, the $\tilde{M}_{x, y, z}$ then depend (in addition to the applied field and the magnetic interactions) on both the magnitude $q$ and the orientation $\theta$ of the scattering vector $\mathbf{q}$ [29]. The derivation of the elastic magnetic neutron scattering cross section can be found, e.g., in the textbook by Squires [30].

In our neutron-data analysis below, we subtract the respective SANS signal at the largest available field of 9.5 T (approach-to-saturation regime; compare Fig. 2) from the measured data at lower fields. This subtraction procedure eliminates the nuclear SANS contribution $\left(\propto|\tilde{N}|^{2}\right)$, which is field independent, and it yields the so-called spinmisalignment SANS cross section $d \Sigma_{M} / d \Omega$, which we display here for simplicity only for the parallel scattering geometry:

$$
\begin{aligned}
\frac{d \Sigma_{M}}{d \Omega}= & \frac{8 \pi^{3}}{V} b_{H}^{2}\left(\Delta\left|\tilde{M}_{x}\right|^{2} \sin ^{2} \theta+\Delta\left|\tilde{M}_{y}\right|^{2} \cos ^{2} \theta\right. \\
& \left.+\Delta\left|\tilde{M}_{z}\right|^{2}+\Delta C T \sin \theta \cos \theta\right),
\end{aligned}
$$

where $\Delta\left|\tilde{M}_{x}\right|^{2}:=\left|\tilde{M}_{x}\right|^{2}(H)-\left|\tilde{M}_{x}\right|^{2}(9.5 \mathrm{~T}$ ) (and so on for the other Fourier coefficients) represents the difference between the value of $\left|\tilde{M}_{x}\right|^{2}$ at the actual field $H$ and the measurement at $9.5 \mathrm{~T}\left[C T:=-\left(\tilde{M}_{x} \tilde{M}_{y}^{*}+\tilde{M}_{x}^{*} \tilde{M}_{y}\right)\right]$. If it is 

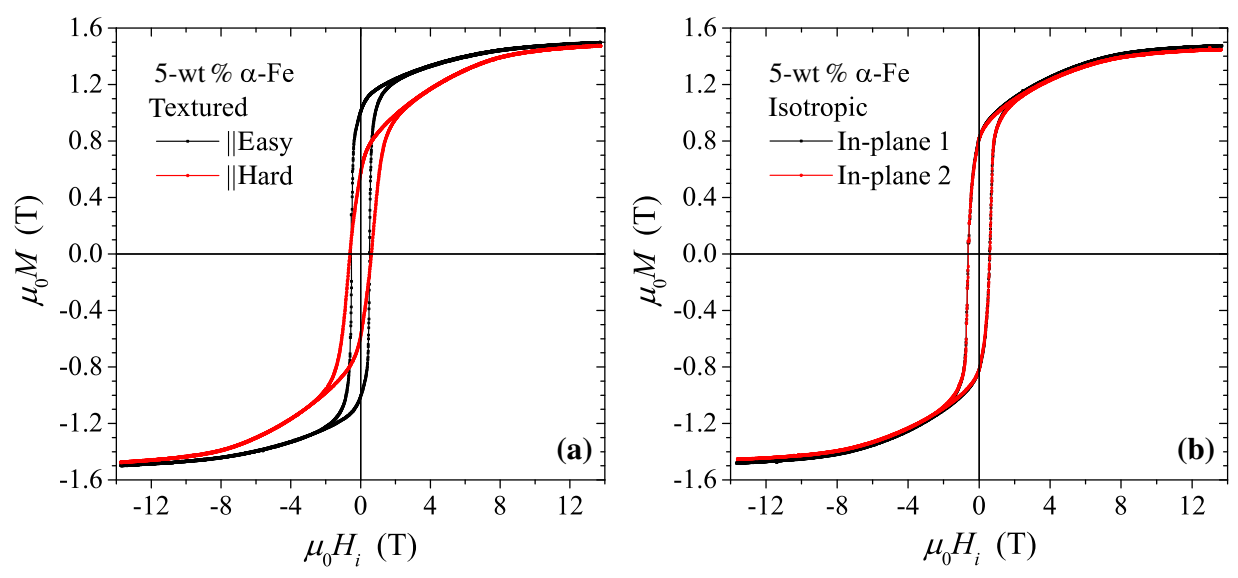

FIG. 2. Room-temperature magnetization curves of textured (a) and isotropic (b) $\mathrm{Nd}_{2} \mathrm{Fe}_{14} \mathrm{~B} / \alpha$-Fe containing, respectively, 5 wt $\%$ of $\alpha$-Fe. Measurements are carried out for the magnetic field applied parallel and perpendicular to the texture axis (pressing direction) in (a) and for two different in-plane directions in (b) (the "in-plane 2" direction is rotated by $90^{\circ}$ with respect to the "in-plane 1" direction). Magnetization data (on the rectangular-shaped samples) are corrected for demagnetizing effects using the magnetometric demagnetizing factor [31]. possible to fully saturate the sample [i.e., $\mathbf{M}(\mathbf{r})=$ $\left.\left\{0,0, M_{z}=M_{s}(\mathbf{r})\right\}\right]$ and if one restricts the considerations (subtraction procedure) to the approach-to-saturation regime, where the field dependence of the longitudinal Fourier component can be neglected [i.e., $\left|\tilde{M}_{z}\right|^{2}(H)-$ $\left|\tilde{M}_{s}\right|^{2} \rightarrow 0$ ], then $d \Sigma_{M} / d \Omega$ (for $\mathbf{k}_{0} \| \mathbf{H}_{0}$ ) reduces to

$$
\begin{aligned}
\frac{d \Sigma_{M}}{d \Omega}= & \frac{8 \pi^{3}}{V} b_{H}^{2}\left(\left|\tilde{M}_{x}\right|^{2} \sin ^{2} \theta+\left|\tilde{M}_{y}\right|^{2} \cos ^{2} \theta\right. \\
& +C T \sin \theta \cos \theta)
\end{aligned}
$$

and likewise for the $\mathbf{k}_{0} \perp \mathbf{H}_{0}$ geometry.

Furthermore, it is decisive for the later discussion to note that for a ferromagnet with a statistically isotropic microstructure, the parallel total (nuclear and magnetic) $d \Sigma / d \Omega$ and $d \Sigma_{M} / d \Omega$ [Eqs. (2) and (3)] are generally isotropic, i.e., $\theta$ independent (see, e.g., Fig. 21 in Ref. [14], Fig. 4 in Ref. [32], or Figs. 5(b), 7(c), and 7(d) below). In other words, although the individual contributions to the parallel SANS cross section are highly anisotropic (e.g., $\left|\tilde{M}_{x}\right|^{2} \sin ^{2} \theta$ ), their corresponding sums in Eqs. (2) and (3) are isotropic for a statistically isotropic ferromagnet; this is not true for the perpendicular geometry [Eq. (1)] in which $d \Sigma_{M} / d \Omega$ generally exhibits a pronounced angular anisotropy, even for a statistically isotropic magnetic material. Based on the results of the magnetization, electron microscopy, x-ray diffraction, and, in particular, the SANS measurements (see below), we see that the condition of statistical isotropy is fulfilled by the isotropic sample (on a wide range of length scales), whereas the hot-deformed specimen exhibits anisotropic physical properties.

The (normalized) correlation function $c(r)$ of the spin misalignment can be computed from azimuthally averaged data via [33]

$$
c(r)=\frac{\int_{0}^{\infty} \frac{d \Sigma_{M}}{d \Omega}(q) J_{0}(q r) q d q}{\int_{0}^{\infty} \frac{d \Sigma_{M}}{d \Omega}(q) q d q},
$$

where $J_{0}(q r)$ denotes the zeroth-order Bessel function. Analysis of $c(r)$ provides information about the characteristic magnetic length scales $[15,16,21]$.

\section{RESULTS AND DISCUSSION}

Figure 2 displays the room-temperature magnetization curves of textured [Fig. 2(a)] and isotropic [Fig. 2(b)] $\mathrm{Nd}_{2} \mathrm{Fe}_{14} \mathrm{~B} / \alpha$-Fe. The coercive fields are $\mu_{0} H_{c}=0.57 \mathrm{~T}$ (textured) and $\mu_{0} H_{c}=0.61 \mathrm{~T}$ (isotropic). The saturation polarization is estimated by extrapolating the data to infinite field: we find $\mu_{0} M_{s}=1.57 \pm 0.01 \mathrm{~T}$ (textured) and $\mu_{0} M_{s}=1.53 \pm 0.01 \mathrm{~T}$ (isotropic) with ensuing remanence-to-saturation ratios of about 0.64 (textured easy), 0.36 (textured hard), and 0.53 (isotropic). Consistent with the magnetization data, the transmission-electron-microscopy images of the textured sample [Figs. 3(a) and 3(b)] reveal a weakly anisotropic microstructure (with a measured aspect ratio of about 1.3), while the isotropic sample [Fig. 3(c)] exhibits equiaxed grains (with a measured aspect ratio of about 1.0). The degree of misorientation of the grains of the textured sample relative to the nominal [001] pressing direction is estimated by analyzing about 50 grains on a focused-ion-beam prepared lamella. We find that the average misorientation angle of the short axis of the grains relative to the pressing direction [" $p$ " in Fig. 3(a)] is $21 \pm 6^{\circ}$.

$\mathrm{X}$-ray-diffraction measurements carried out (in transmission mode) at the ALBA Synchrotron (see Fig. 4) unambiguously prove the presence of a texture along the (horizontal) pressing direction; specifically, diffraction peaks of the type $(00 l)$ do present two maxima around $\theta=0^{\circ}$ and $\theta=180^{\circ}$ in the Debye-Scherrer rings [see Fig. 4(d)]. Additionally, we find evidence of the presence of texture along other crystallographic directions; peaks of the type $(h k 0)$ exhibit two maxima around $90^{\circ}$ and $270^{\circ}$ [see Fig. 4(c)]. This latter observation is of relevance when discussing the results of the magnetic neutron-data analysis (see below). We also perform a Rietveld analysis of the diffraction data of both samples [data sets obtained from the 

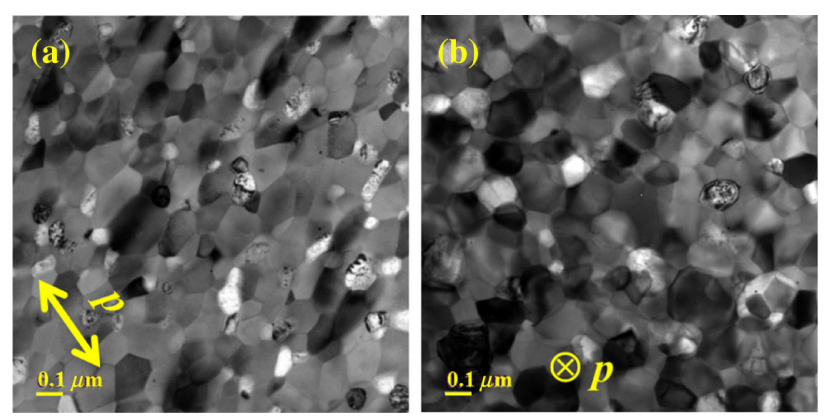

FIG. 3. Bright-field transmissionelectron-microscopy images of the textured [(a) and (b)] and isotropic (c) $\mathrm{Nd}_{2} \mathrm{Fe}_{14} \mathrm{~B} / \alpha$-Fe nanocomposites (5-wt \% Fe). The average sizes of the anisotropic grains of the textured sample are estimated [from (a) and (b)] as, respectively, approximately $110 \mathrm{~nm}$ (parallel to the pressing direction $p$ ) and approximately $140 \mathrm{~nm}$ (perpendicular to the pressing direction $p$ ), while the average grain diameter of the isotropic sample is approximately $20 \mathrm{~nm}$. complete diffraction patterns shown in Figs. 4(a) and 4(b)]. Both data sets can be refined with the same phases $\left[\mathrm{Nd}_{2} \mathrm{Fe}_{14} \mathrm{~B}\left(P 4_{2} / m n m\right.\right.$ space group $)$ and $\alpha$-Fe]. The structures obtained from the analysis of the $\mathrm{Nd}_{2} \mathrm{Fe}_{14} \mathrm{~B}$ phases of the isotropic and textured samples do not show significant differences. Note that the refinements do not improve when varying the occupation of $\mathrm{Nd}$ atoms; likewise, there is no indication of the presence of additional phases in the diffraction patterns.

Figure 5 depicts (for $\mathbf{k}_{0} \| \mathbf{H}_{0}$ ) the two-dimensional unpolarized total SANS scattering cross sections $d \Sigma / d \Omega$ of the textured and isotropic Nd-Fe-B-based nanocomposites at selected applied magnetic fields $(9.5 \mathrm{~T}$, remanence, coercive field). The isotropic sample [Fig. 5(b)] exhibits an
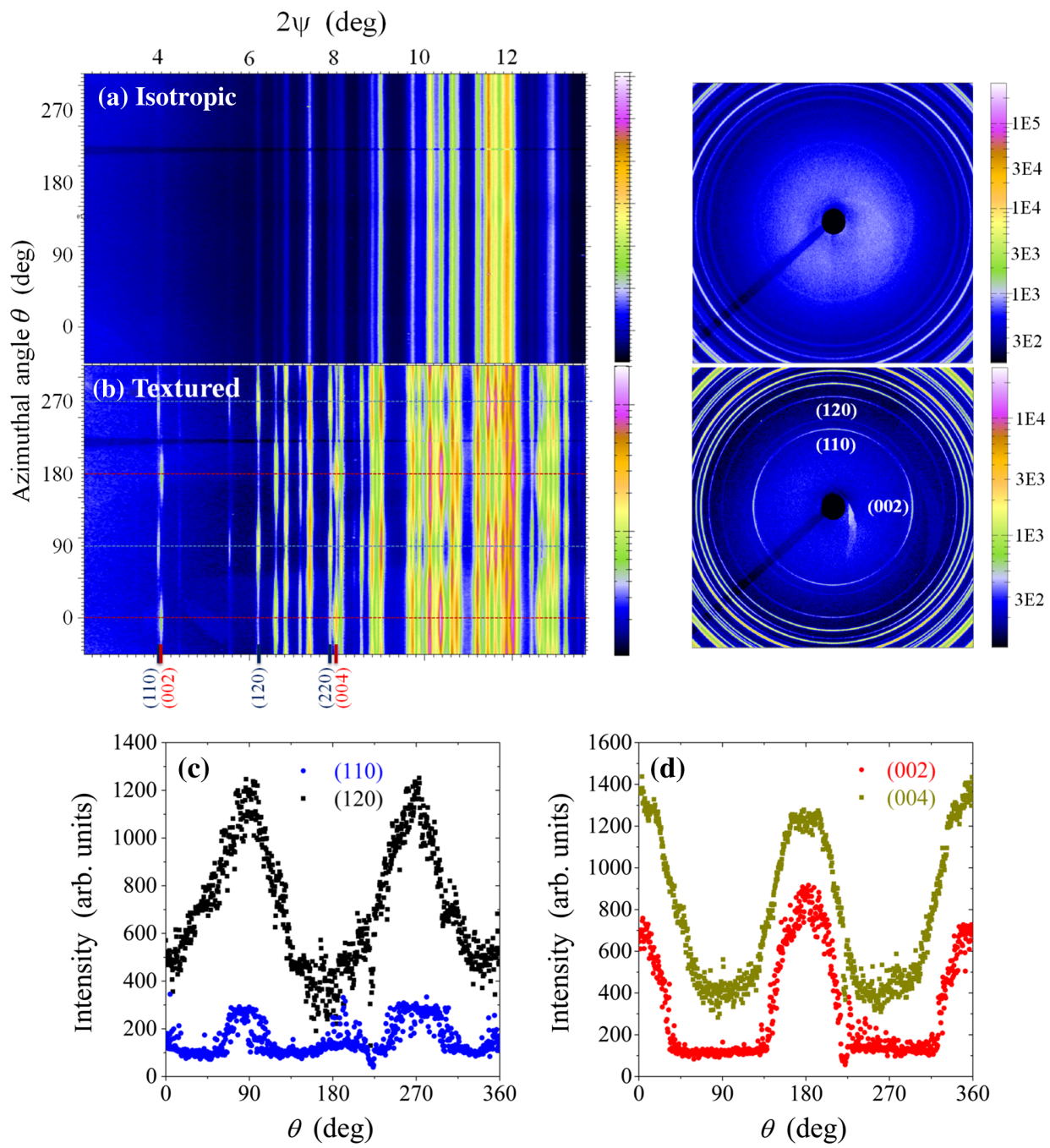

FIG. 4. Synchrotron x-ray diffraction data of the isotropic (a) and textured (b) $\mathrm{Nd}_{2} \mathrm{Fe}_{14} \mathrm{~B} / \alpha$-Fe nanocomposites $(5-\mathrm{wt} \% \mathrm{Fe}) \quad\left(\lambda_{\mathrm{XRD}}=\right.$ $0.4246 \AA$ ). The pressing direction of the hot-deformed sample is horizontal (same scattering geometry as in the neutron experiment; compare Fig. 1). Left images in (a),(b): Integrated intensity as a function of azimuthal angle $\theta$ and scattering angle $2 \psi$. Right images in (a),(b): Corresponding Debye-Scherrer diffraction rings (logarithmic color scale). Radial integration of synchrotron data is performed with the Fit2D software [34]. (c),(d) $\theta$ dependence of the $\mathrm{x}$-ray intensities of the (110) and (120) peaks and of the (002) and (004) peaks. 

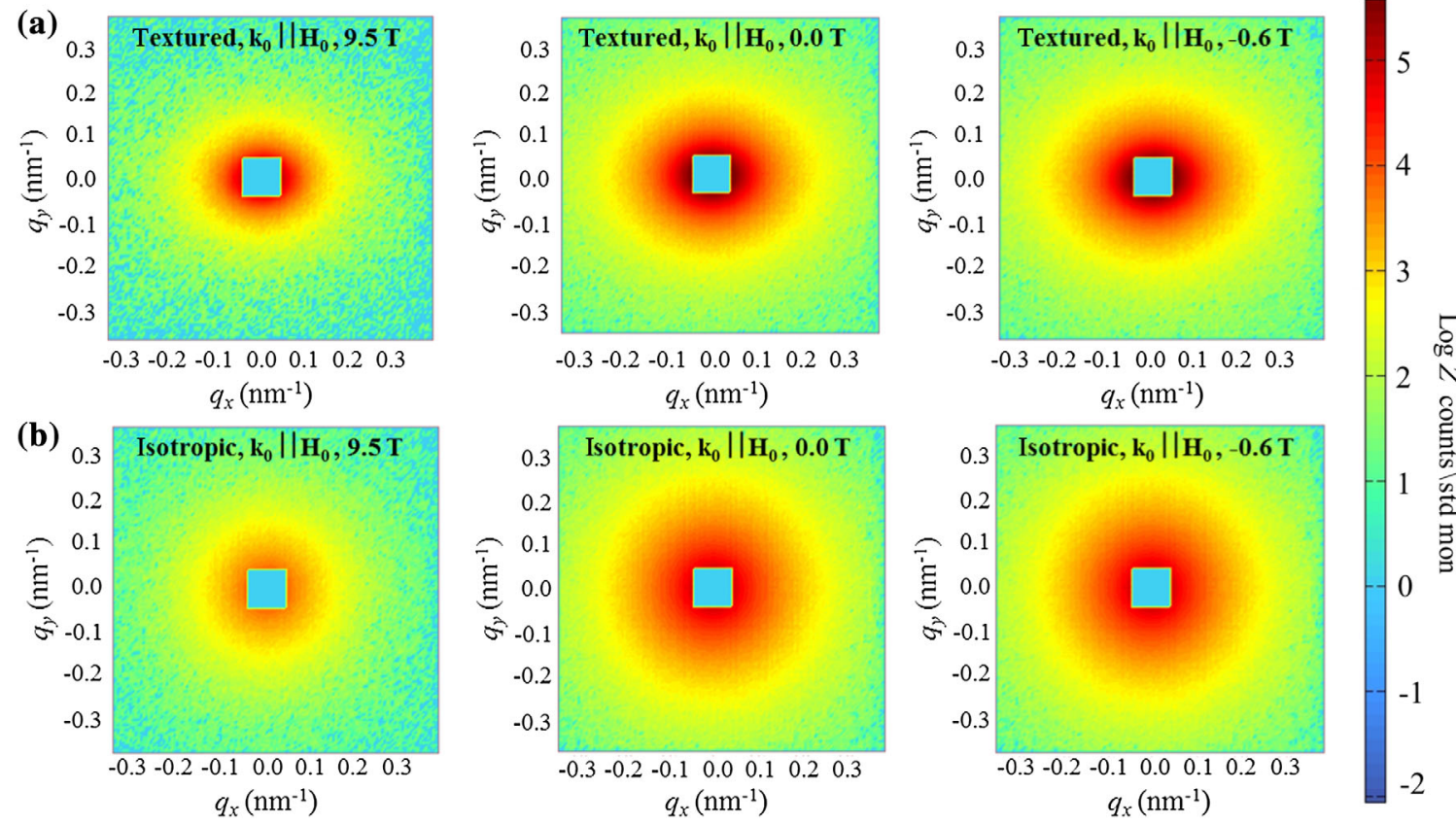

FIG. 5. Color-coded two-dimensional intensity maps of the total unpolarized $d \Sigma / d \Omega$ in the plane perpendicular to the incoming neutron beam at selected applied magnetic fields (see insets) (logarithmic color scale; counts per standard monitor) $\left(\mathbf{k}_{0} \| \mathbf{H}_{0}\right)$. $d \Sigma / d \Omega$ of the textured (a) and isotropic (b) $\mathrm{Nd}_{2} \mathrm{Fe}_{14} \mathrm{~B} / \alpha$-Fe nanocomposite. $\mathbf{H}_{0}$ is normal to the detector plane.

isotropic scattering pattern at all fields investigated, whereas the textured sample [Fig. 5(a)] shows anisotropic scattering with an elongation along the horizontal direction. The corresponding (over $2 \pi$ ) azimuthally averaged data sets are displayed in Fig. 6; between the coercive field and the largest available field of $9.5 \mathrm{~T}$, the cross section of the isotropic sample changes (roughly) by about an order of magnitude at the smallest momentum transfers $q$ (and about half an order of magnitude for the textured sample).

While the textured nanocomposite reveals a power-lawtype scattering over most of the $q$ range, the isotropic sample exhibits a more structured $d \Sigma / d \Omega$ with significant curvature at lower and medium $q$. This difference in $d \Sigma / d \Omega$ is most likely related to the difference in the average grain sizes and the ensuing magnetization fluctuations on a nanometer length scale: the isotropic sample has an average grain size of approximately $20 \mathrm{~nm}$, while the textured $\mathrm{Nd}-\mathrm{Fe}-\mathrm{B}$ possesses a larger particle size of the order of $100 \mathrm{~nm}$ (compare the TEM images in Fig. 3). We also note that the $d \Sigma / d \Omega$ of both samples (data not shown) as well as the spin-misalignment SANS cross section $d \Sigma_{M} / d \Omega$ [see Fig. 6(c)] are characterized by power-law exponents $n$ that are larger than 4 . This is in agreement with the notion of spin-misalignment scattering, i.e., scattering due to canted
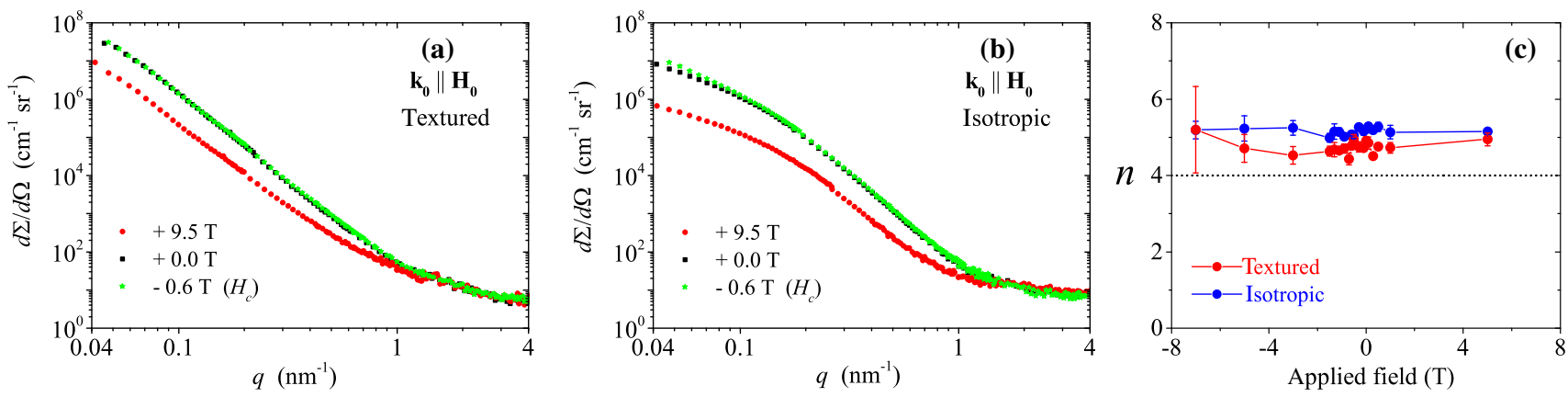

FIG. 6. Azimuthally averaged total unpolarized SANS cross sections $d \Sigma / d \Omega$ at selected applied magnetic fields (log-log scale) $\left(\mathbf{k}_{0} \| \mathbf{H}_{0}\right) . d \Sigma / d \Omega$ of the textured (a) and isotropic (b) $\mathrm{Nd}_{2} \mathrm{Fe}_{14} \mathrm{~B} / \alpha$-Fe nanocomposite. (c) Applied-field dependence of the power-law exponent $n$ in $d \Sigma_{M} / d \Omega=K / q^{n}$ for the textured and isotropic $\mathrm{Nd}_{2} \mathrm{Fe}_{14} \mathrm{~B} / \alpha$-Fe nanocomposite. $d \Sigma_{M} / d \Omega$ is obtained by subtracting, respectively, the total $d \Sigma / d \Omega$ at $9.5 \mathrm{~T}$; the fits are restricted to the interval $0.4 \mathrm{~nm}^{-1} \lesssim q \lesssim 0.6 \mathrm{~nm}^{-1}$. The choice of this particular interval is motivated by the condition that $q \xi \gg 1$, where $\xi$ is some characteristic length scale in the system. We also try other intervals (both smaller and larger), and the results are qualitatively and quantitatively similar. Dotted horizontal line $(n=4)$ corresponds to scattering due to sharp interfaces (Porod) or to exponentially correlated magnetization fluctuations. 
spins with a characteristic magnetic-field-dependent wavelength [14]. It is also quite obvious from this observation that the corresponding magnetization fluctuations in real space are not exponentially correlated (see Fig. 8 below).

As discussed previously, for $\mathbf{k}_{0} \| \mathbf{H}_{0}$, any anisotropy of $d \Sigma / d \Omega$ (or of $d \Sigma_{M} / d \Omega$ ) is indicative of an anisotropic microstructure. At magnetic saturation, the total SANS signal arises from nanoscale spatial fluctuations in the nuclear density and in the saturation magnetization $M_{s}(\mathbf{r})$, presumably at internal $\mathrm{Nd}_{2} \mathrm{Fe}_{14} \mathrm{~B} / \alpha$-Fe interfaces. The nuclear-scattering-length density contrast between the $\mathrm{Nd}_{2} \mathrm{Fe}_{14} \mathrm{~B}$ phase and the $\alpha$-Fe phase amounts to $\Delta \rho_{\text {nuc }} \cong 1.63 \times 10^{14} \mathrm{~m}^{-2}$, whereas, at saturation, the magnetic contrast can be estimated as $\Delta \rho_{\text {mag }}=b_{H} \Delta M \cong$ $1.37 \times 10^{14} \mathrm{~m}^{-2}$, where $\Delta M$ denotes the difference in saturation magnetization between $\alpha$-Fe $\left(\mu_{0} M_{s}=2.2 \mathrm{~T}\right)$ and $\mathrm{Nd}_{2} \mathrm{Fe}_{14} \mathrm{~B}\left(\mu_{0} M_{s}=1.61 \mathrm{~T}\right)$. By assuming that the elements of the microstructure which give rise to nuclear scattering $|\tilde{N}|^{2}$ are identical to those which give rise to longitudinal magnetic scattering $b_{H}^{2}\left|\tilde{M}_{z}\right|^{2}$, one finds for a saturated sample that the ratio of nuclear-to-magnetic SANS equals $|\tilde{N}|^{2} /\left(b_{H}^{2}\left|\tilde{M}_{z}\right|^{2}\right) \cong 1.42$. With reference to the electron-microscopy results (Fig. 3), which reveal a (weakly) anisotropic grain shape (aspect ratio of approximately 1.3), it is then obvious that a (weakly) horizontally elongated SANS pattern can already be generated at saturation by the combined action of the nuclear and longitudinal magnetic form factors.

Subtracting the total $d \Sigma / d \Omega$ at $9.5 \mathrm{~T}$ from the total $d \Sigma / d \Omega$ at lower fields, we obtain the spin-misalignment SANS cross section $d \Sigma_{M} / d \Omega$ [Eq. (3)], which is free of the nuclear SANS. We remind the reader that in the parallel scattering geometry, $d \Sigma_{M} / d \Omega$ is expected to be isotropic for a microstructure which exhibits statistical isotropy and that this is not true for the perpendicular geometry. This can be understood by taking into account that for $\mathbf{k}_{0}\left\|\mathbf{H}_{0}\right\| \mathbf{e}_{z}$ both transversal-magnetization components $M_{x}$ and $M_{y}$ are equally distributed in the detector plane [compare Fig. 1(b)], whereas for $\mathbf{k}_{0} \perp \mathbf{H}_{0}$ [compare Fig. 1(a)], only one of the transversal-magnetization components $\left(M_{y}\right)$ lies in the plane that is spanned by the detector coordinates, while the other one $\left(M_{x}\right)$ is along the incident beam. Hence, for a statistically isotropic microstructure, the symmetry (asymmetry) of the transversal spin components then explains the isotropy (anisotropy) of the detector pattern which is observed in the parallel (perpendicular) scattering geometry. Based on these considerations, it becomes clear that for a magnetically textured sample in the parallel geometry, $d \Sigma_{M} / d \Omega$ is expected to be anisotropic. Indeed, the results for $d \Sigma_{M} / d \Omega$ for the textured nanocomposite [Figs. 7(a) and 7(b)] still reveal an angular anisotropy with maxima parallel and antiparallel to the horizontal texture axis. Inspection of Eq. (3) then suggests that this observation may be due to (i) spin components which are directed along the $\pm \mathbf{e}_{y}$ direction [cf. the term $\Delta\left|\tilde{M}_{y}\right|^{2} \cos ^{2} \theta$
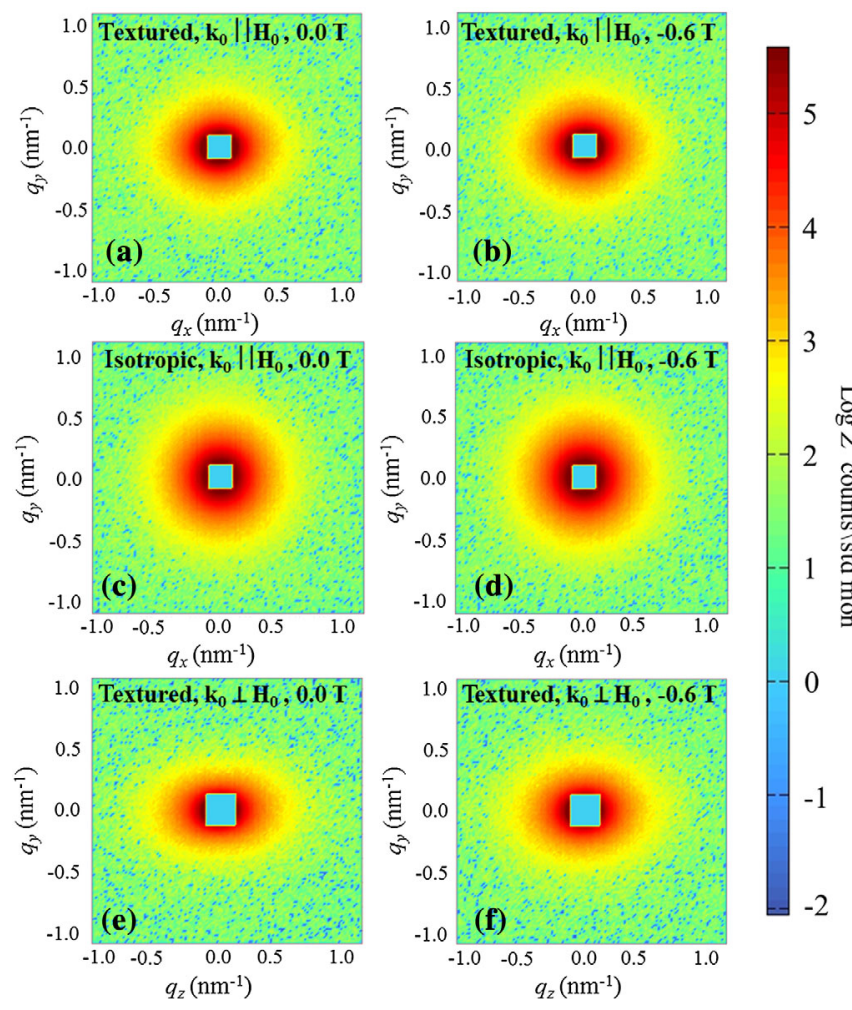

FIG. 7. Selected results for the spin-misalignment SANS cross section $d \Sigma_{M} / d \Omega$ of the textured and isotropic $\mathrm{Nd}_{2} \mathrm{Fe}_{14} \mathrm{~B} / \alpha-\mathrm{Fe}$ nanocomposite for $\mathbf{k}_{0} \| \mathbf{H}_{0}[(\mathrm{a})-(\mathrm{d})]$ and for $\mathbf{k}_{0} \perp \mathbf{H}_{0}[(\mathrm{e}),(\mathrm{f})]$ (logarithmic color scale). The respective data set at the maximum applied field of $9.5 \mathrm{~T}$ is subtracted. In (a)-(d), $\mathbf{H}_{0}$ is normal to the detector plane, whereas in (e) and (f), $\mathbf{H}_{0}$ is horizontal in the plane.

in Eq. (3)] and/or due to (ii) the particle form-factor anisotropy [cf. the term $\Delta\left|\tilde{M}_{z}\right|^{2}$ in Eq. (3)]. However, the measurements in the $\mathbf{k}_{0} \perp \mathbf{H}_{0}$ geometry [compare Fig. 1(a)] suggest that longitudinal magnetization fluctuations play only a minor role: if the $d \Sigma_{M} / d \Omega$ (for $\mathbf{k}_{0} \perp \mathbf{H}_{0}$ ) are dominated by $\Delta\left|\tilde{M}_{z}\right|^{2}$, a $\sin ^{2} \theta$-type anisotropy with intensity maxima along the vertical direction results [compare Eq. (1)]. This is, however, not visible in the experimental data [Figs. 7(e) and 7(f)], which exhibit a horizontal elongation [cf. the term $\left|\tilde{M}_{y}\right|^{2} \cos ^{2} \theta$ in Eq. (1)]. In other words, the anisotropy of the scattering pattern for $\mathbf{k}_{0} \| \mathbf{H}_{0}$ [Figs. 7(a) and 7(b)] is due to an anisotropy in the magnetic microstructure not to the form-factor anisotropy $\Delta\left|\tilde{M}_{z}\right|^{2}$ of the particles.

We emphasize that although the mean magnetization in the remanent state is directed along the $c$ axis $\left[\mathbf{e}_{z}\right.$ direction in Fig. 1(a) and $\mathbf{e}_{x}$ direction in Fig. 1(b)], the magnetic neutron scattering cross section is in both geometries dominated by the respective $\left|\tilde{M}_{y}\right|^{2} \cos ^{2} \theta$ term, which (in real space) is related to small misaligned spin components varying along the $\pm \mathbf{e}_{y}$ direction [35]. This anisotropy in the spin microstructure may be related to the finding of a 


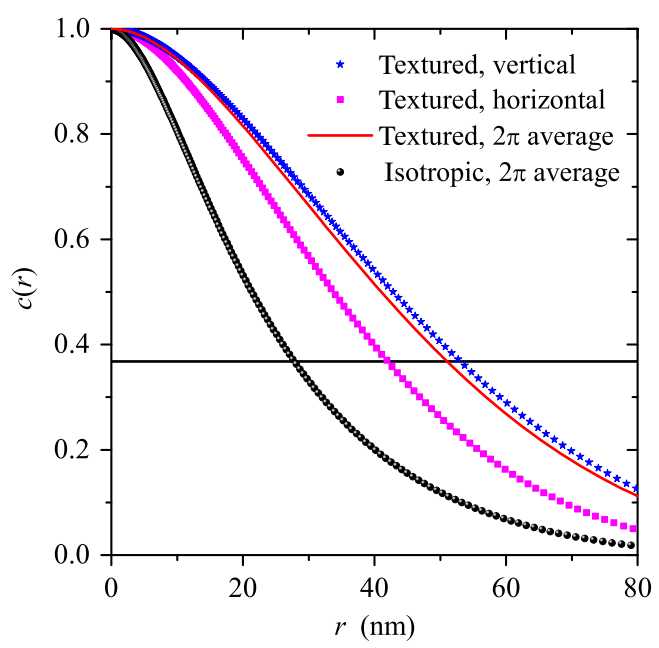

FIG. 8. Normalized correlation function $c(r)$ of the spin misalignment [Eq. (5)] for the textured and isotropic $\mathrm{Nd}_{2} \mathrm{Fe}_{14} \mathrm{~B} / \alpha$-Fe nanocomposite in the remanent state. $c(r)$ of the textured sample is computed using $d \Sigma_{M} / d \Omega$ averaged along the vertical and horizontal directions $\left( \pm 7.5^{\circ}\right.$ sector averages $)$ as well as using the full circular $(2 \pi)$ average of $d \Sigma_{M} / d \Omega$; the $c(r)$ of the isotropic sample is computed using the corresponding $2 \pi$-averaged $d \Sigma_{M} / d \Omega$ (see inset). Solid horizontal line: $c(r)=\exp (-1)$. The physically relevant information content of $c(r)$ is restricted to the interval $\left[r_{\min }, r_{\max }\right]$ with approximately $r_{\text {min }} \cong 2 \pi / q_{\max }=2 \mathrm{~nm}$ and $r_{\text {max }} \cong 2 \pi / q_{\min }=130 \mathrm{~nm}$.

crystallographic texture: as shown in Fig. 4, diffraction peaks of the type $(h k 0)$ exhibit two maxima along the vertical direction $\left(\theta=90^{\circ}\right.$ and $\left.\theta=270^{\circ}\right)$. In this context, we emphasize that the recent electron-microscopy and three-dimensional atom-probe tomography work by Liu et al. [36] reports anisotropic properties of the grain-boundary phase in hot-deformed nanocrystalline $\mathrm{Nd}-\mathrm{Fe}-\mathrm{B}$ magnets; namely, these authors found that the concentration of rare-earth elements is higher for intergranular phases parallel to the flat surface of the plateletshaped $\mathrm{Nd}_{2} \mathrm{Fe}_{14} \mathrm{~B}$ grains as compared to intergranular phases along the short side of the platelets. It is, therefore, of interest to combine magnetic SANS with atom-probe tomography in order to see whether the anisotropy of the concentration profile at grain boundaries is related to the presence of a spin texture. This might be important, since from the technological point of view one may certainly want to avoid such a spin texture in order to maintain a high remanent magnetization. The investigation of the relation between the crystallographic texture and the spin texture is of interest in its own right and beyond the scope of this paper; we believe that its understanding is key for elucidating the potential of Nd-Fe-B nanocomposites for further technological applications.

The characteristic size of the spin inhomogeneities can be estimated by computing [using Eq. (5)] the correlation function $c(r)$ of the spin misalignment. Figure 8 depicts the correlation functions for the isotropic as well as for the textured sample; the $c(r)$ are computed using data sets for $d \Sigma_{M} / d \Omega$ which are obtained by either carrying out a full circular $(2 \pi)$ average of $d \Sigma_{M} / d \Omega$ or by taking an average along selected directions in $q$ space, e.g., along the horizontal and vertical directions in the detector plane. From the initial decay of $c(r)$, the correlation length $l_{C}$ of the spin misalignment can be deduced. As the value for $l_{C}$ we take the $\exp (-1)$ length; we emphasize that this does not imply that the correlations decay exponentially. The correlation length $l_{C}$ is a measure for the range over which perturbations in the spin microstructure-around a particular lattice defect-are transmitted by the exchange interaction into the surrounding crystal lattice [33]. For a polycrystalline bulk ferromagnet containing a large amount of different imperfections, the experimental value for $l_{C}$ represents a weighted average over the different defects. As discussed in the Introduction, for the textured Nd-Fe-Bbased nanocomposite, possible origins for magnetization inhomogeneities are related, e.g., to the presence of crystal defects introduced via hot pressing or to the jump in the magnetic materials parameters (exchange constant, direction and magnitude of magnetic anisotropy, and saturation magnetization) at the grain-boundary regions. An analysis of the correlation functions (in the remanent state) yields for the textured specimen $l_{C} \cong 53 \mathrm{~nm}$ along the vertical direction and $l_{C} \cong 42 \mathrm{~nm}$ along the horizontal direction; these values are smaller than the average particle size, which is estimated by TEM (see Fig. 3; average grain thickness, approximately $110 \mathrm{~nm}$; average grain diameter, approximately $140 \mathrm{~nm}$ ). For the isotropic nanocomposite, $l_{C} \cong 28 \mathrm{~nm}$, which is of the order of the average particle size $D \cong 20 \mathrm{~nm}$. Hence, the finding that $l_{C} \cong 40-50 \mathrm{~nm}$ for the hot-deformed sample suggests the existence of spin disorder, i.e., gradients in the magnetization distribution on an intraparticle length scale. Compatible with Ref. [36], these results (anisotropy of $l_{C}$ ) indicate that the microscopic nature of the lattice defects (e.g., the $\mathrm{Nd}_{2} \mathrm{Fe}_{14} \mathrm{~B} / \alpha$-Fe interfaces) along these two directions are different (as is manifest by the different correlation lengths). In this respect, field-dependent SANS measurements are helpful, since they allow one to determine the field evolution of $l_{C}$, from which the size of the defect (causing the spin perturbation) and the exchange-correlation length may be obtained $[15,16]$.

\section{CONCLUSION}

Using magnetic SANS, we provide a comparative study of the magnetic microstructure of textured and isotropic $\mathrm{Nd}_{2} \mathrm{Fe}_{14} \mathrm{~B} / \alpha$-Fe nanocomposites. Our neutron-data analysis suggests that the spin-misalignment scattering of the textured sample is dominated by spin components along one direction perpendicular to the easy $c$ axis (pressing direction) of the $\mathrm{Nd}_{2} \mathrm{Fe}_{14} \mathrm{~B}$ grains. This anisotropy in the magnetization distribution is accompanied by the presence of a crystallographic texture along these directions. 
Possible origins for the spin canting (on an intraparticle length scale) are discussed and are related to the presence of perturbed interface regions, crystalline imperfections, and/ or a dispersion in the orientation distribution of the easy $c$ axes. In agreement with the $\mathrm{x}$-ray synchrotron and neutron data, we find anisotropic real-space correlations, with a correlation length that is estimated at about $40-50 \mathrm{~nm}$ in the remanent state. The results demonstrate the power of magnetic SANS for analyzing anisotropic magnetic structures on a nanometer length scale; in particular, the complementary use of the perpendicular and parallel scattering geometries (for the textured sample) provides results that are otherwise not accessible with only one geometry. Moreover, the presented methodology is quite generally applicable to $\mathrm{Nd}-\mathrm{Fe}-\mathrm{B}$ magnets, and future neutron work will address the role of the intergranular Nd-rich layers for the coercivity mechanism of sintered magnets.

\section{ACKNOWLEDGMENTS}

D. M. acknowledges financial support from the National Research Fund of Luxembourg (Grant No. INTER/DFG/ 12/07). This paper is based on results obtained from the future pioneering program "Development of Magnetic Material Technology for High-Efficiency Motors" commissioned by the New Energy and Industrial Technology Development Organization. The neutron experiments are performed at the Swiss spallation neutron source SINQ, Paul Scherrer Institute, Villigen, Switzerland. The ALBA Synchrotron is acknowledged for the provision of beam time. We thank Birgit Heiland (INM, Saarbrücken) and Jörg Schmauch (Universität des Saarlandes) for the electron-microscopy work.

[1] R. Coehoorn, D. B. De Mooij, J. P. W. B. Duchateau, and K. H.J. Buschow, Novel permanent magnetic materials made by rapid quenching, J. Phys. (Paris), Colloq. 49, C8-669 (1988).

[2] H. A. Davies, K. J. A. Mawella, R. A. Buckley, G. E. Carr, A. Manaf, and A. Jha, in Concerted European Action on Magnets (CEAM), edited by I. V. Mitchell, J. M. D. Coey, D. Givord, I. R. Harris, and R. Hanitsch (Springer Netherlands, Dordrecht, 1989), pp. 543-557.

[3] O. Gutfleisch, A. Bollero, A. Handstein, D. Hinz, A. Kirchner, A. Yan, K.-H. Müller, and L. Schultz, Nanocrystalline high performance permanent magnets, J. Magn. Magn. Mater. 242-245, 1277 (2002).

[4] O. Gutfleisch, M. A. Willard, E. Brück, C. H. Chen, S. G. Sankar, and J. Ping Liu, Magnetic materials and devices for the 21st century: Stronger, lighter, and more energy efficient, Adv. Mater. 23, 821 (2011).

[5] J. P. Liu, in Nanoscale Magnetic Materials and Applications, edited by J. P. Liu, E. Fullerton, O. Gutfleisch, and D. J. Sellmyer (Springer, New York, 2009), pp. 309-335.
[6] S. Bance, H. Oezelt, T. Schrefl, M. Winklhofer, G. Hrkac, G. Zimanyi, O. Gutfleisch, R. F. L. Evans, R. W. Chantrell, T. Shoji, M. Yano, N. Sakuma, A. Kato, and A. Manabe, High energy product in Battenberg structured magnets, Appl. Phys. Lett. 105, 192401 (2014).

[7] J. Liu, H. Sepehri-Amin, T. Ohkubo, K. Hioki, A. Hattori, T. Schrefl, and K. Hono, Effect of $\mathrm{Nd}$ content on the microstructure and coercivity of hot-deformed Nd-Fe-B permanent magnets, Acta Mater. 61, 5387 (2013).

[8] H. Sepehri-Amin, T. Ohkubo, S. Nagashima, M. Yano, T. Shoji, A. Kato, T. Schrefl, and K. Hono, High-coercivity ultrafine-grained anisotropic Nd-Fe-B magnets processed by hot deformation and the $\mathrm{Nd}-\mathrm{Cu}$ grain boundary diffusion process, Acta Mater. 61, 6622 (2013).

[9] N. Umetsu, A. Sakuma, and Yuta Toga, First principles study on interfacial electronic structures in exchange-spring magnets, Phys. Rev. B 93, 014408 (2016).

[10] Daisuke Ogawa, Kunihiro Koike, Shigemi Mizukami, Takamichi Miyazaki, Mikihiko Oogane, Yasuo Ando, and Hiroaki Kato, Negative exchange coupling in $\mathrm{Nd}_{2} \mathrm{Fe}_{14} \mathrm{~B}(100) / \alpha-\mathrm{Fe}$ interface, Appl. Phys. Lett. 107, 102406 (2015).

[11] O. Gutfleisch, Controlling the properties of high energy density permanent magnetic materials by different processing routes, J. Phys. D 33, R157 (2000).

[12] A. Kirchner, J. Thomas, O. Gutfleisch, D. Hinz, K.-H. Müller, and L. Schultz, HRTEM studies of grain boundaries in die-upset Nd-Fe-Co-Ga-B magnets, J. Alloys Compd. 365, 286 (2004).

[13] T. G. Woodcock, Y. Zhang, G. Hrkac, G. Ciuta, N. M. Dempsey, T. Schrefl, O. Gutfleisch, and D. Givord, Understanding the microstructure and coercivity of high performance NdFeB-based magnets, Scr. Mater. 67, 536 (2012).

[14] A. Michels, Magnetic small-angle neutron scattering of bulk ferromagnets, J. Phys. Condens. Matter 26, 383201 (2014).

[15] J.-P. Bick, D. Honecker, F. Döbrich, K. Suzuki, E. P. Gilbert, H. Frielinghaus, J. Kohlbrecher, J. Gavilano, E. M. Forgan, R. Schweins, P. Lindner, R. Birringer, and A. Michels, Magnetization reversal in Nd-Fe-B based nanocomposites as seen by magnetic small-angle neutron scattering, Appl. Phys. Lett. 102, 022415 (2013).

[16] E. A. Périgo, E. P. Gilbert, and A. Michels, Magnetic SANS study of a sintered Nd-Fe-B magnet: Estimation of defect size, Acta Mater. 87, 142 (2015).

[17] J.-P. Bick, K. Suzuki, E. P. Gilbert, E. M. Forgan, R. Schweins, P. Lindner, C. Kübel, and A. Michels, Exchangestiffness constant of a Nd-Fe-B based nanocomposite determined by magnetic neutron scattering, Appl. Phys. Lett. 103, 122402 (2013).

[18] É. A. Périgo, E. P. Gilbert, K. L. Metlov, and A. Michels, Experimental observation of magnetic poles inside bulk magnets via $\mathbf{q} \neq 0$ Fourier modes of magnetostatic field, New J. Phys. 16, 123031 (2014).

[19] Tetsuro Ueno, Kotaro Saito, Masao Yano, Masaaki Ito, Tetsuya Shoji, Noritsugu Sakuma, Akira Kato, Akira Manabe, Ai Hashimoto, Elliot P. Gilbert, Uwe Keiderling, and Kanta Ono, Multiple magnetic scattering in small-angle neutron scattering of Nd-Fe-B nanocrystalline magnet, Sci. Rep. 6, 28167 (2016). 
[20] E. A. Périgo, D. Mettus, E. P. Gilbert, P. Hautle, N. Niketic, B. van den Brandt, J. Kohlbrecher, P. McGuiness, Z. Fu, and A. Michels, Magnetic microstructure of a textured Nd-Fe-B sintered magnet characterized by small-angle neutron scattering, J. Alloys Compd. 661, 110 (2016).

[21] E. A. Périgo, I. Titov, R. Weber, D. Honecker, E. P. Gilbert, M. F. De Campos, and A. Michels, Small-angle neutron scattering study of coercivity enhancement in grainboundary-diffused Nd-Fe-B sintered magnets, J. Alloys Compd. 677, 139 (2016).

[22] M. Yano, K. Ono, A. Manabe, N. Miyamoto, T. Shoji, A. Kato, Y. Kaneko, M. Harada, H. Nozaki, and J. Kohlbrecher, Magnetic reversal observation in nano-crystalline Nd-Fe-B magnet by SANS, IEEE Trans. Magn. 48, 2804 (2012).

[23] M. Yano, K. Ono, M. Harada, A. Manabe, T. Shoji, A. Kato, and J. Kohlbrecher, Investigation of coercivity mechanism in hot deformed Nd-Fe-B permanent magnets by small-angle neutron scattering, J. Appl. Phys. 115, 17A730 (2014).

[24] K. Saito, T. Ueno, M. Yano, M. Harada, T. Shoji, N. Sakuma, A. Manabe, A. Kato, U. Keiderling, and K. Ono, Magnetization reversal of a $\mathrm{Nd}-\mathrm{Cu}$-infiltrated $\mathrm{Nd}-\mathrm{Fe}-\mathrm{B}$ nanocrystalline magnet observed with small-angle neutron scattering, J. Appl. Phys. 117, 17B302 (2015).

[25] J. Kohlbrecher and W. Wagner, The new SANS instrument at the Swiss spallation source SINQ, J. Appl. Crystallogr. 33, 804 (2000).

[26] N. Niketic, B. van den Brandt, W. Th. Wenckebach, J. Kohlbrecher, and P. Hautle, Polarization analysis in neutron small-angle scattering with a novel triplet dynamic nuclear polarization spin filter, J. Appl. Crystallogr. 48, 1514 (2015).

[27] www.ill.eu/lss/grasp.
[28] François Fauth, Roeland Boer, Fernando Gil-Ortiz, Catalin Popescu, Oriol Vallcorba, Inma Peral, Daniel Fullà, Jordi Benach, and Jordi Juanhuix, The crystallography stations at the Alba synchrotron, Eur. Phys. J. Plus 130, 160 (2015).

[29] Sergey Erokhin, Dmitry Berkov, and Andreas Michels, Dipolar spin-misalignment correlations in inhomogeneous magnets: Comparison between neutron scattering and micromagnetic approaches, Phys. Rev. B 92, 014427 (2015).

[30] G. L. Squires, Introduction to the Theory of Thermal Neutron Scattering (Dover Publications, New York, 1978).

[31] A. Aharoni, Demagnetizing factors for rectangular ferromagnetic prisms, J. Appl. Phys. 83, 3432 (1998).

[32] A. Michels, S. Erokhin, D. Berkov, and N. Gorn, Micromagnetic simulation of magnetic small-angle neutron scattering from two-phase nanocomposites, J. Magn. Magn. Mater. 350, 55 (2014).

[33] D. Mettus and A. Michels, Small-angle neutron scattering correlation functions of bulk magnetic materials, J. Appl. Crystallogr. 48, 1437 (2015).

[34] A. P. Hammersley, S. O. Svensson, M. Hanfland, A. N. Fitch, and D. Hausermann, Two-dimensional detector software: From real detector to idealised image or two-theta scan, High Press. Res. 14, 235 (1996).

[35] A. Michels, R. N. Viswanath, and J. Weissmüller, Domain formation and long-range spin disorder in Vitroperm, Europhys. Lett. 64, 43 (2003).

[36] J. Liu, H. Sepehri-Amin, T. Ohkubo, K. Hioki, A. Hattori, and K. Hono, Microstructure evolution of hot-deformed Nd-Fe-B anisotropic magnets, J. Appl. Phys. 115, 17A744 (2014). 